\title{
Mutation density changes in SARS-CoV-2 are related to the pandemic stage but to a lesser extent in the dominant strain with mutations in spike and RdRp
}

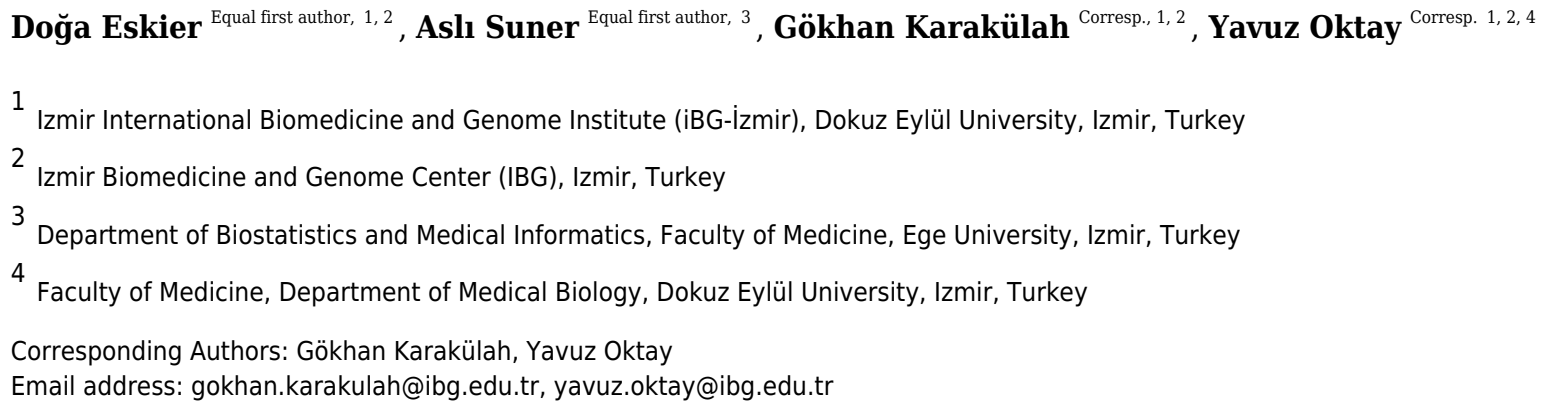

Since its emergence in Wuhan, China in late 2019, the origin and evolution of SARS-CoV-2 have been among the most debated issues related to COVID-19. Throughout its spread around the world, the viral genome continued acquiring new mutations and some of them became widespread. Among them, 14408 C>T and 23403 A>G mutations in RdRp and S, respectively, became dominant in Europe and the US, which led to debates regarding their effects on the mutability and transmissibility of the virus. In this study, we aimed to investigate possible differences between time-dependent variation of mutation densities (MDe) of viral strains that carry these two mutations and those that do not. Our analyses at the genome and gene level led to two important findings: First, time-dependent changes in the average MDe of circulating SARS-CoV-2 genomes showed different characteristics before and after the beginning of April, when daily new case numbers started levelling off. Second, this pattern was much delayed or even non-existent for the "mutant" (MT) strain that harbored both 14408 C>T and 23403 A>G mutations. Although these differences were not limited to a few hotspots, it is intriguing that the MDe increase is most evident in two critical genes, $\mathrm{S}$ and Orflab, which are also the genes that harbor the defining mutations of the MT genotype. The nature of these unexpected relationships warrants further research. 
1 Mutation density changes in SARS-CoV-2 are related to the pandemic stage

2 but to a lesser extent in the dominant strain with mutations in spike and

3 RdRp

4

5 Doğa Eskier ${ }^{1,2, ~ *}$, Aslı Suner ${ }^{3, ~ *}$, Gökhan Karakülah ${ }^{1,2, \dagger}$, Yavuz Oktay ${ }^{1,2,4, \dagger}$

$7 \quad{ }^{1}$ Izmir Biomedicine and Genome Center (IBG), Izmir, Turkey

8 2Izmir International Biomedicine and Genome Institute, Dokuz Eylül University, Izmir, Turkey

$9{ }^{3}$ Department of Biostatistics and Medical Informatics, Faculty of Medicine, Ege University,

10 Izmir, Turkey

$11{ }^{4}$ Faculty of Medicine, Department of Medical Biology, Dokuz Eylül University, Izmir, Turkey

12 *These authors contributed equally to the work presented here and should therefore be regarded as

13 first authors.

15 Co-corresponding authors:

16 Gökhan Karakülah, E-mail: gokhan.karakulah@deu.edu.tr

17 Yavuz Oktay, E-mail: yavuz.oktay@ibg.edu.tr 
19 Abstract

20 Since its emergence in Wuhan, China in late 2019, the origin and evolution of SARS-CoV-2 have

21 been among the most debated issues related to COVID-19. Throughout its spread around the

22 world, the viral genome continued acquiring new mutations and some of them became widespread.

23 Among them, $14408 \mathrm{C}>\mathrm{T}$ and $23403 \mathrm{~A}>\mathrm{G}$ mutations in RdRp and $\mathrm{S}$, respectively, became

24 dominant in Europe and the US, which led to debates regarding their effects on the mutability and

25 transmissibility of the virus. In this study, we aimed to investigate possible differences between

26 time-dependent variation of mutation densities (MDe) of viral strains that carry these two

27 mutations and those that do not. Our analyses at the genome and gene level led to two important

28 findings: First, time-dependent changes in the average MDe of circulating SARS-CoV-2 genomes

29 showed different characteristics before and after the beginning of April, when daily new case

30 numbers started levelling off. Second, this pattern was much delayed or even non-existent for the

31 "mutant" (MT) strain that harbored both $14408 \mathrm{C}>\mathrm{T}$ and $23403 \mathrm{~A}>\mathrm{G}$ mutations. Although these

32 differences were not limited to a few hotspots, it is intriguing that the MDe increase is most evident

33 in two critical genes, S and Orflab, which are also the genes that harbor the defining mutations of

34 the MT genotype. The nature of these unexpected relationships warrants further research.

35 Keywords: SARS-CoV-2, COVID-19, Surface glycoprotein, Spike, RNA-dependent RNA

36 polymerase, RdRp, mutation density

\section{Introduction}

38 COVID-19 (coronavirus disease 2019) is an ongoing pandemic that has been observed in

$397,553,182$ patients and responsible for 423,349 deaths as of 13 June 2020. It is characterized by

40 respiratory system problems and slow onset fever, and is caused by SARS-CoV-2, a novel 
41 betacoronavirus of presumably zoonotic origin. It has first been identified in the Hubei province

42 of China in December 2019, with confirmed human transmission in January 2020 (Chan et al.,

43 2020; Riou \& Althaus, 2020), and is currently a global concern. COVID-19 has high

44 transmissibility (D’Arienzo \& Coniglio, 2020; Petersen \& Gökengin, 2020), a capacity of

45 asymptomatic cases to spread the disease (Wong et al., 2020), and poses a high degree of danger

46 to both vulnerable individuals and the healthcare systems via such widespread and invisible

47 transmission (Liu et al., 2020; Basu, 2020, p.). Therefore, despite the disease having a current

48 mortality rate of $<6 \%$, lower than similar diseases caused by betacoronaviruses such as SARS or

49 MERS (Zhang \& Holmes, 2020), a fuller understanding of and cure for the underlying pathogen

50 is a high priority for researchers and clinicians everywhere.

51 Soon after its spread to Europe and the US, a strain of SARS-CoV-2 with two non-synonymous

52 mutations in the RNA-dependent RNA polymerase (RdRp) and Spike (S) proteins, namely

$5314408 \mathrm{C}>\mathrm{T}(\mathrm{P} 323 \mathrm{~L})$ and $23403 \mathrm{~A}>\mathrm{G}(\mathrm{D} 614 \mathrm{G})$ became the dominant form particularly in Europe

54 and to some extent in the US, as well. What makes these two mutations particularly interesting is

55 the critical functions of the $\mathrm{RdRp}$ and $\mathrm{S}$ proteins: $\mathrm{RdRp}$ is the main protein responsible for

56 replication of the viral RNA, while S mediates the binding of viral particles to human cells that

57 express the angiotensin converting enzyme-2 (ACE2) protein, and thereby facilitates viral entry.

58 Claims of increased transmissibility of this new strain due to spike D614G mutation by Korber et

59 al. was met with caution, as other factors such as founder effect, drift etc. could be responsible

60 for its dominance, as well (Korber et al., 2020). Our previous study suggested that RdRp 14408

$61 \mathrm{C}>\mathrm{T}$ mutation is associated with SARS-CoV-2 genome evolution and could even be working

62 synergistically with the $23403 \mathrm{~A}>\mathrm{G}$ (D614G) mutation (Eskier et al., 2020). Although these and

63 other studies provided suggestive evidence that indeed this new strain could have altered 
64 phenotypic characteristics (Lorenzo-Redondo et al., 2020; Wagner et al., 2020), only after

65 mechanistic studies are performed in cell culture and animal models, we will be able to know

66 whether these inferences are true (Grubaugh, Hanage \& Rasmussen, 2020). In this respect, a

67 series of reports from several labs has lent support to the increased transmissibility hypothesis for

68 viruses carrying the $23403 \mathrm{~A}>\mathrm{G}$ mutation: pseudotyped lenti- and retro-viruses with the mutant

69 spike protein infect human cells in culture more efficiently, compared to those with the wild-type

70 spike (Zhang et al., 2020; Ozono et al., 2020; Yurkovetskiy et al., 2020; Hu et al., 2020;

71 Daniloski et al., 2020). However, the underlying mechanism is less clear: Zhang et al. suggested

72 increased stability of the spike protein to explain increased infectivity of the mutant viruses,

73 while Daniloski et al. suggested the mutant protein is more resistant to cleavage. Other studies

74 also pointed to the importance of the spike protein for the unique properties of the SARS-CoV-2

75 (Walls et al., 2020; Cheng et al., 2020). However, animal studies will be required to further test

76 the effects of the D614G mutation to reach a more definitive conclusion. Nevertheless, many

77 lines of evidence support increased transmissibility hypothesis and therefore, spike mutations

78 should probably be monitored closely and analyzed in-depth.

79 In our previous study, where we analyzed 11,208 SARS-CoV-2 genome sequences, we showed

80 that RdRp mutations, particularly $14408 \mathrm{C}>\mathrm{T}$ mutation, were associated with SARS-CoV-2

81 genome evolution and higher mutation density (Eskier et al., 2020). In the current study, we

82 analyzed the time-dependent changes in the mutation densities of several SARS-CoV-2 genes

83 and asked whether the patterns of change were different between the strain with the $14408 \mathrm{C}>\mathrm{T} /$

$8423403 \mathrm{~A}>\mathrm{G}$ mutations compared to those with neither mutation. 


\section{Materials and Methods}

86 Genome sequence filtering, retrieval, and preprocessing

87 SARS-CoV-2 isolate genome sequences were obtained from the GISAID EpiCoV database and 88 their variants were annotated as previously described ("GISAID Initiative"; Eskier et al., 2020).

89 Briefly, the sequences obtained on 24 May 2020 were filtered for full length genomes with high

90 coverage and to remove environmental or non-human host samples. The remaining genomes

91 were edited to mask low-quality base calls and aligned against the reference SARS-CoV-2

92 genome using the MAFFT multiple sequence alignment program. Variant sites were retrieved

93 using snp-sites, and their effect on peptide sequences were annotated using the ANNOVAR suite

94 of tools. Due to the low quality and gap-heavy content in a majority of isolates, the 5'

95 untranslated regions (1-265) and the 100 nucleotides at the 3 ' end were also removed from

96 analysis. In addition, we removed isolate genomes without well-defined time of sequencing or

97 geographical location data, to ensure the time and location variables can be clearly associated

98 with the mutations, for a final count of 19,705 genomes.

99 Mutation density calculation

100 The annotated variants were separated into synonymous and nonsynonymous groups, with

101 variants in nucleotides not located in gene loci considered synonymous. Gene mutation densities

102 were calculated separately for synonymous and nonsynonymous mutations, by dividing the

103 number of variant sites in the gene locus of an isolate by the length of the locus. Densities for

104 each full open reading frame, including the surface glycoprotein (S), envelope glycoprotein (E),

105 nucleocapsid phosphoprotein $(\mathrm{N})$, and membrane glycoprotein $(\mathrm{M})$ genes were calculated, as

106 well as the RdRp coding region of ORF1ab and the whole genome. 


\section{Statistical Analysis}

108 The categorical variables were analyzed with frequency tables and descriptive statistics were

109 used for the continuous variables. Shapiro-Wilk normality test was used to examine whether the

110 numeric values had a normal distribution in groups. When the numeric values were not normally

111 distributed, Mann-Whitney U test was used to compare the median values of two independent

112 groups. The level of significance was chosen as 0.05 in all hypothesis tests. IBM SPSS Version

11325.0 statistical package was used for statistical analyses.

\section{Results and Discussion}

\section{$11514408 \mathrm{C}>\mathrm{T}$ and $23403 \mathrm{~A}>\mathrm{G}$ mutations are associated with mutation density increase over}

\section{6 time}

117 In order to determine whether the SARS-CoV-2 strain with $14408 \mathrm{C}>\mathrm{T} / 23403 \mathrm{~A}>\mathrm{G}$ mutations

118 was different from those that carried neither mutation, with respect to time-dependent changes in

119 mutation density, first, we determined the number of synonymous and non-synonymous

120 mutations. The number of sites carrying mutations, after the low-quality filters were applied to

121 isolates and the 5' and 3' ends, was 7870, with 16563 potential variants. 11095 of these variants

122 were characterized as synonymous, as they did not alter any known peptide sequence, while

1235468 of them were non-synonymous, as they either changed amino acid residues in the translated

124 proteins, or altered the protein length by changing start or stop codon locations. To eliminate the

125 bias that would be caused by the $14408 \mathrm{C}>\mathrm{T}$ and $23403 \mathrm{~A}>\mathrm{G}$ mutations on the nonsynonymous

126 mutation density of isolates they were present in, which would be substantial considering their

127 high frequency, these two nucleotides were also masked during calculations of non-synonymous

128 mutation density. 
129 For further analysis, we focused on the two countries with the highest number of isolates

130 sequenced, the United Kingdom (UK) and the United States (US), which in total contributed

$13158.76 \%$ of all isolates that passed the quality filters. Furthermore, we focused only on isolates

132 that were sequenced after the first genome with both $14408 \mathrm{C}>\mathrm{T}$ and $23403 \mathrm{~A}>\mathrm{G}$ mutations were

133 identified in the respective geographic regions (26 February 2020 for UK, 28 February 2020 for

134 US). Isolates that carried both of the mutations were named "mutant", or "MT" in short, and

135 those that were wild-type at both positions were named "wild-type", or "WT in short; few

136 isolates that carried only one of the mutations was excluded. In total, the isolates were separated

137 into four categories: UK-WT (1708 genomes), UK-MT (4903 genomes), US-WT (1683

138 genomes), and US-MT (3407 genomes). There was a total of 6163 variants found in isolates in

139 these categories, with 3533 of them being nonsynonymous, across 5693 polymorphic sites.

140 We calculated the "average mutation density per day per genome" (hereafter referred as mutation

141 density, or MDe for short) for both synonymous and non-synonymous mutations in all four

142 categories, as well as the correlation of MDe with time, using Spearman correlation (Fig. 1). We

143 identified a strong positive correlation between non-synonymous MDe and time in both UK and

144 US in MT samples $(\rho=0.70$, p-value $<0.001)$, however, a much weaker correlation was

145 observed in WT samples $(\rho=0.27, p$-value $=0.002)$, indicating a potential relationship between

146 non-synonymous mutations and $14408 \mathrm{C}>\mathrm{T} / 23403 \mathrm{~A}>\mathrm{G}$ genotype. A similar correlation was

147 identified for synonymous mutations in MT samples, albeit weaker than for non-synonymous

148 mutations $(\rho=0.64, p$-value $<0.001)$. No significant correlation was identified for synonymous

149 mutations in WT samples $(\rho=0.15, p$-value $=0.09)$. One possible explanation for this tight

150 correlation is that MT genomes accumulate both synonymous and non-synonymous mutations

151 over time, whereas WT strains show more variation in their mutation accumulation rate. 
152 However, closer inspection of the plots points to further differences between MT and WT SARS-

153 CoV-2 genomes: Rather than a monotonic change in MDe over time, certain time points seem to

154 be critical milestones at which the mutation densities take sharp turns. One of them is roughly

155 Day 100, which is defined as the $100^{\text {th }}$ day following the first SARS-CoV-2 genome sequenced,

156 and corresponds to 2 April 2020 (Fig. 1). Whereas the MDe of WT strains keep increasing until

157 Day 100, for both synonymous and non-synonymous, this trend is disrupted from there on and

158 actually a slight decrease is observed. On the other hand, albeit a slight reduction in the strength

159 of correlation, there is almost no change in the rate of increase in MDe (both synonymous and

160 non-synonymous) for the MT genomes, only a slight decrease after Day 130 is observed. Of

161 note, the synonymous MDe of MT genomes starts increasing after Day 80, compared to the non-

162 synonymous MDe, which shows a steadier increase starting from the beginning.

163 The next obvious question is, "Why are there such sharp changes in MDe at certain timepoints,

164 particularly around Day 100 for WT and Day 80 for MT?" Even though a simple answer is

165 unlikely, we looked into epidemiological data for clues. Interestingly, Day 80 (13 March 2020)

166 corresponds to the beginning of a 20-day period when daily case numbers started increasing

167 dramatically in both the UK and the US, and also is the day "national emergency" was declared

168 in the US; Day 100 (2 April 2020) roughly corresponds to the end of this period (Dong, Du \&

169 Gardner, 2020) (Figure S1) and the daily case numbers show a slow but steady decline after Day

170100 in both countries. It makes sense that both strains accumulated more and more mutations as

171 they spread fast between Days 80-100. However, it is less clear why this trend continued only in

172 the MT strain after Day 100, when the strict control measures slowed the spreading of SARS-

173 CoV-2. 
174 Next, we determined how MT and WT strains differ at gene level, in terms of MDe. To do so,

175 we examined the overall distribution of mutations combined for the previously specified time

176 period and compared them between the MT and WT genomes (Figs. S2-3, Table S1). Only 2

$177(\mathrm{RdRp}, \mathrm{N})$ of the 12 genes showed consistent differential synonymous MDe in the MT genotype

178 compared to the WT genotype, for UK and US. RdRp MDe was lower in the MT genotype

179 (Mean Rank: 104.91 vs. 40.30, $\mathrm{p}<0.001$ in UK; 80.26 vs. 52.38 , p-value $<0.001$ in US), whereas

180 MDe of N gene was higher in the MT genotype (Mean Rank: 37.21 vs. 101.59, p-value $<0.001$

181 in UK; 54.05 vs. 77.41, p-value $<0.001$ in US). Although differences were significant in the S

182 gene, it was in the opposite directions, higher in UK (Mean Rank: 61.13 vs. 79.93, p=0.006)

183 and lower in US (Mean Rank: 81.17 vs. 51.51, p-value < 0.001), for the MT genotype. A closer

184 look pointed to country-specific differences: While only 3 of 12 genes showed differences in

185 non-synonymous MDe between MT and WT genotypes for UK, this number was much higher

186 for US, 9 of 12 genes showed differential non-synonymous MDe. This marked difference

187 between the two countries is likely due to the differences in the composition of the WT strains,

188 which has much higher variety in the US compared to UK owing to heavier influence of Asian

189 SARS-CoV-2 genomes and multiple independent lines of viral spread around the US (Worobey

190 et al., 2020; Deng et al., 2020). Indeed, such differences are expected, even in the absence of

191 multiple founders, and justify our approach of comparing results of two countries to reach a

192 consensus.

193 As non-synonymous mutations are subject to stronger selection than synonymous mutations and

194 could have different patterns, we also determined possible differences in their MDe values

195 between MT and WT SARS-CoV-2 genomes. Only 2 (S, N) of 12 genes showed consistent

196 differences between the two genotypes in both countries (Table S1). MDe values in both the S 
197 (Mean Rank: 48.88 vs. 91.03, p-value $<0.001$ in UK; 58.95 vs. 72.73, $\mathrm{p}=0.035$ in US) and $\mathrm{N}$

198 genes (Mean Rank: 34.45 vs. 104.09, p-value $<0.001$ in UK; 55.52 vs. 76.01, $\mathrm{p}=0.002$ in US)

199 were higher in the MT genotype. 3 genes (Orflab, Orf3a, Orf6) also showed differences in both

200 countries, however, in the opposite directions. In contrast to the synonymous mutations, higher

201 number of genes had significant differences between non-synonymous MDe values in UK,

202 compared to US (4 vs 2; RdRp, M, E, Orf7b vs Orf8, Orf10); interestingly, MDe values of the

203 MT genotype were higher in the first four and lower in the last two, compared to WT. Overall,

204 MDe of MT and WT genotypes at gene level showed gene-specific and country-specific

205 differences, while only $\mathrm{N}$ gene had consistent differences for both synonymous and non-

206 synonymous mutations in both UK and US.

\section{Non-synonymous mutation densities increase in later samples}

208 After characterization of MDe for the time period that MT genotype existed in the UK or US, we

209 next tested whether genome-level differences between MT and WT genotypes in terms of time

210 dependent changes in MDe are also present at gene level. Therefore, we aimed to understand

211 whether those differences at genome level are merely due to possible founder effects, or due to

212 differences between MT and WT strains' accumulation of mutations over time. Since Day 100

213 appeared to be a turning point, we divided the SARS-CoV-2 genomes into two groups by the

214 time of isolation: early (days 60-100) and late periods (days 101-140), and examined the

215 relationships between the average MDe of the two groups for each gene and country (Figs. 2-3,

216 Table S2). While none of the 12 genes in the WT genotype showed any significant difference in

217 MDe between the early and late periods consistently between UK and US, it was the opposite for

218 the MT genotype: S, RdRp, M, Orflab, and Orf3a genes showed increased MDe in the late

219 period compared to the early period. It was particularly evident for the S and Orflab genes, as 
220 both synonymous and non-synonymous MDe in both genes were significantly increased in the

221 late period in both countries. Interestingly, M gene showed a consistent decrease in the non-

222 synonymous MDe only in the MT genomes, against the common trend of increased MDe in this

223 genotype; however, no such association was observed for the synonymous MDe, it was even the

224 opposite in the UK-MT isolates. On the other hand, the RdRp non-synonymous MDe was

225 increased significantly in the late period in UK-MT isolates, and trended towards increase in US-

226 MT isolates (Mean Rank: 29.76 vs. 38.64, p-value $=0.058$ ); RdRp non-synonymous MDe was

227 also increased in late period UK-MT isolates, however, there was no such increase in the US

228 isolates, consistent with time-independent MDe values (Figure S2), and with the marginally

229 insignificant increase in synonymous MDe in the same isolates. Among changes that were

230 significant for a single-country, there was an overall tendency towards increased MDe in MT

231 genomes, but the opposite was true for WT genomes: 12/14 of single-country changes in MT

232 genomes were associated with increased MDe, whereas 12/16 of such changes in WT genomes

233 were associated with decreased MDe in different genes. These findings suggest that the steady

234 increase in MDe specific to MT isolates even after Day 100 cannot simply be explained by

235 founder effects, or linkage. One could speculate that at least some of the WT strains accumulated

236 mutations that reduced their fitness under lock-down conditions and therefore led to their

237 elimination in the late period (101-140 days), whereas the MT strain was fit enough to keep most

238 or all mutations and even add new ones in this period. However, almost equal contribution of

239 synonymous and non-synonymous mutations may point to other factors in addition to possible

240 differences in fitness. Although S and Orflab genes seem to be the main drivers of the observed

241 genome-wide increase the MDe of MT genomes, it seems that other genes are also contributing

242 to this trend. 
243 Isolates with 14408 C $>$ T \& 23403 A $>$ G mutations (MT genotype) show steady increase in

\section{4 mutations in both $\mathbf{S}$ and RdRp over time}

245 To further investigate whether the shift in MDe happens due to a few distinct sites that are linked

246 with the MT mutations, and could possibly directly affect the host adaptation process, or whether

247 it is caused by a variety of mutations that spread widely both temporally and spatially in the

248 genome, we further divided the early and late time periods into a total of four periods $(60-80,81$ -

$249100,101-120,121-140$ ), and analyzed the distributions of mutations in the S and RdRp coding

250 regions in these four time periods in a pool containing both UK and US isolates (Figs. 4, S4-5).

251 To do so, we calculated the number of mutations in 100-nucleotide bins per isolate sharing a

252 genotype (MT or WT) in that each time period, and observed their distributions, divided into two

253 groups as synonymous and non-synonymous mutations. For the periods 60-80, 81-100, 101-120,

254 and 121-140, the MT / WT distributions were 515 / 622, 4044 / 2105, 3119 / 610, and 632 / 54,

255 respectively. We can see that in the 60-80 day period, the distribution of mutations across the

256 regions were largely comparable between WT and MT genomes, with the exception of the

257 synonymous $14805 \mathrm{C}>\mathrm{T}$ mutation in the RdRp, which is exclusive to WT isolates (Fig. 4A). The

258 ratio of mutations in MT to WT steadily increase over time in both synonymous and non-

259 synonymous categories across UK and US isolates (Figs. S4-5), with MT genome mutations

260 more visible in most bins in the 121-140 day period, with the major exception of the $14805 \mathrm{C}>\mathrm{T}$

261 mutation being preserved in WT isolates (Fig. 4B). 100-nucleotide binned totals and the mutated

262 isolate ratios for all sites in the regions are available in sheet 1 and 2 of Supplementary File,

263 respectively. These observations suggest that the increase in MDe in MT isolates is due to

264 overall increased number of nucleotides in the genome becoming mutated over time, rather than

265 increased frequency of certain genotypes with higher MDe. Although it is unclear whether the 
$26614805 \mathrm{C}>\mathrm{T}$ synonymous mutation has any stabilizing effect on the WT MDe, as the mutation is

267 found only 1698 samples in all isolates that passed our quality filters, including non-UK and

268 non-US isolates, and 1106 of those isolates are UK-WT and US-WT isolates, its high frequency

269 in WT isolates is more likely to be the result of a founder effect, rather than any selection.

\section{Conclusions}

271 Earlier studies of ours, as well as others, pointed at the emergence of RdRp and S mutations and

272 their spread across the world as the pandemic progressed (Pachetti et al., 2020; Eskier et al.,

273 2020). These reports provided evidence supporting possible effects of dominant mutations on

274 SARS-CoV-2 genome evolution. In this study, we reveal an unexpected relationship between the

275 SARS-CoV-2 mutation densities and viral transmission dynamics at population level in humans.

276 While the average mutation densities increased steadily by time during the fast-spreading period

277 of COVID-19 in both countries, this trend ended around Day 100 (2 April 2020), when daily new

278 case numbers started reaching a plateau. It seems, however, that not all strains of SARS-CoV-2

279 are affected equally by the epidemiological factors (i.e. counter-measures that led to reduced

280 daily new case numbers in UK and US, host factors, treatment regimens, etc.), in terms of

281 mutation densities. SARS-CoV-2 strain with both RdRp $14408 \mathrm{C}>\mathrm{T}$ and S $23403 \mathrm{~A}>\mathrm{G}$

282 mutations, which became dominant in Europe and US first, differed and continued accumulating

283 both synonymous and non-synonymous mutations after Day 100, particularly in the S and Orfla

284 genes. Therefore, two related questions remain to be answered: 1) Is there any causal relationship

285 between mutation density changes and viral transmission in population? If the answer is 'yes',

286 what is the nature of this relationship? 2) What is the biological basis of differences between the

287 'mutant' and 'wild-type' strains, if there is any? It is intriguing that the main drivers of the 
288 genome-level increase in MDe were two critical genes, S and Orfla, which are also the genes

289 that harbor the defining mutations of the MT genotype.

290 It should also be noted that the MDe of the MT genomes started showing signs of decline after

291 Day 130 (2 May 2020), although no obvious change in the epidemiological data is seen around

292 this date. Further studies are warranted that will investigate the relationship between mutations

293 and the clinical phenotypes, as more data will become widely available.

294 In conclusion, we propose that monitoring the average mutation densities over time and relating

295 to the epidemiological and clinical data may help establish genotype/phenotype relations, and

296 possibly predict the increases and decreases in new cases.

\section{Additional Information and Declarations}

298 Acknowledgements

299 The authors would like to thank Mr. Alirıza Arıbaş from Izmir Biomedicine and Genome Center

300 for his technical assistance. The authors also would like to extend their thanks to Izmir

301 Biomedicine and Genome Center (IBG) COVID19 platform IBG- COVID19 for their support in

302 implementing the study and the Scientific and Technological Research Council of Turkey

303 (TUBITAK) for their financial support of IBG-COVID19.

\section{Funding}

305 YO is supported by the Turkish Academy of Sciences Young Investigator Program (TÜBA-

306 GEBİP).

\section{Grant Disclosures}

308 Not applicable. 
309 Competing Interests

310 AS and GK are associate editors in PeerJ.

\section{Author Contributions}

312 - DE conceived and designed the experiments, performed the experiments, analyzed the data,

313 contributed reagents/materials/analysis tools, prepared figures and/or tables, authored or

314 reviewed drafts of the paper, approved the final draft.

315 - AS conceived and designed the experiments, performed the experiments, analyzed the data,

316 contributed reagents/materials/analysis tools, prepared figures and/or tables, authored or

317 reviewed drafts of the paper, approved the final draft.

318 - GK conceived and designed the experiments, performed the experiments, analyzed the data,

319 contributed reagents/materials/analysis tools, prepared figures and/or tables, authored or

320 reviewed drafts of the paper, approved the final draft.

321 -YO conceived and designed the experiments, performed the experiments, analyzed the data,

322 contributed reagents/materials/analysis tools, prepared figures and/or tables, authored or

323 reviewed drafts of the paper, approved the final draft.

\section{Supplemental Information}

325 Supplemental materials are included with this research.

326 Data Availability

327 The processed genotyping VCF file is available here: doi:10.17632/jv87xwj7fv.1

\section{References}

329 Basu S. 2020. Non-communicable disease management in vulnerable patients during Covid-19. Indian Journal of Medical Ethics V:103-105. DOI: 10.20529/IJME.2020.041. 
331 Chan JF-W, Yuan S, Kok K-H, To KK-W, Chu H, Yang J, Xing F, Liu J, Yip CC-Y, Poon RW-

332

333

334

335

336

337

338

339

340

341

342

343

344

345

346

347

348

349

350

351

352

353

S, Tsoi H-W, Lo SK-F, Chan K-H, Poon VK-M, Chan W-M, Ip JD, Cai J-P, Cheng VC-

C, Chen H, Hui CK-M, Yuen K-Y. 2020. A familial cluster of pneumonia associated with the 2019 novel coronavirus indicating person-to-person transmission: a study of a family cluster. Lancet (London, England) 395:514-523. DOI: 10.1016/S0140-6736(20)30154-9.

Cheng MH, Zhang S, Porritt RA, Arditi M, Bahar I. 2020. An insertion unique to SARS-CoV-2 exhibits superantigenic character strengthened by recent mutations. bioRxiv. DOI: 10.1101/2020.05.21.109272.

Daniloski Z, Jordan TX, Ilmain JK, Guo X, Bhabha G, tenOever BR, Sanjana NE. 2020. The Spike D614G mutation increases SARS-CoV-2 infection of multiple human cell types. bioRxiv:2020.06.14.151357. DOI: 10.1101/2020.06.14.151357.

D'Arienzo M, Coniglio A. 2020. Assessment of the SARS-CoV-2 basic reproduction number, R0, based on the early phase of COVID-19 outbreak in Italy. Biosafety and Health. DOI: 10.1016/j.bsheal.2020.03.004.

Deng X, Gu W, Federman S, du Plessis L, Pybus OG, Faria N, Wang C, Yu G, Bushnell B, Pan C-Y, Guevara H, Sotomayor-Gonzalez A, Zorn K, Gopez A, Servellita V, Hsu E, Miller S, Bedford T, Greninger AL, Roychoudhury P, Starita LM, Famulare M, Chu HY, Shendure J, Jerome KR, Anderson C, Gangavarapu K, Zeller M, Spencer E, Andersen KG, MacCannell D, Paden CR, Li Y, Zhang J, Tong S, Armstrong G, Morrow S, Willis M, Matyas BT, Mase S, Kasirye O, Park M, Masinde G, Chan C, Yu AT, Chai SJ, Villarino E, Bonin B, Wadford DA, Chiu CY. 2020. Genomic surveillance reveals multiple introductions of SARS-CoV-2 into Northern California. Science:eabb9263. DOI: 10.1126/science.abb9263.

Peer) reviewing PDF | (2020:06:50041:1:0:CHECK 17 Jul 2020) 
354 Dong E, Du H, Gardner L. 2020. An interactive web-based dashboard to track COVID-19 in real

355

356

357

358

359

360

361

362

363

364

365

366

367

368

369

370

371

372

373

374

375 time. The Lancet Infectious Diseases 20:533-534. DOI: 10.1016/S1473-3099(20)30120-

Eskier D, Karakülah G, Suner A, Oktay Y. 2020. RdRp mutations are associated with SARSCoV-2 genome evolution. bioRxiv:2020.05.20.104885. DOI: 10.1101/2020.05.20.104885.

GISAID Initiative. Available at https://www.epicov.org/epi3/frontend\#272e13 (accessed May 19, 2020).

Grubaugh ND, Hanage WP, Rasmussen AL. 2020. Making sense of mutation: what D614G means for the COVID-19 pandemic remains unclear. Cell. DOI: 10.1016/j.cell.2020.06.040.

Hu J, He C-L, Gao Q-Z, Zhang G-J, Cao X-X, Long Q-X, Deng H-J, Huang L-Y, Chen J, Wang K, Tang N, Huang A-L. 2020. D614G mutation of SARS-CoV-2 spike protein enhances viral infectivity. bioRxiv:2020.06.20.161323. DOI: 10.1101/2020.06.20.161323.

Korber B, Fischer WM, Gnanakaran S, Yoon H, Theiler J, Abfalterer W, Foley B, Giorgi EE, Bhattacharya T, Parker MD, Partridge DG, Evans CM, Freeman TM, Silva T de, Group on behalf of the SC-19 G, LaBranche CC, Montefiori DC. 2020. Spike mutation pipeline reveals the emergence of a more transmissible form of SARS-CoV-2. bioRxiv:2020.04.29.069054. DOI: 10.1101/2020.04.29.069054.

Liu K, Chen Y, Lin R, Han K. 2020. Clinical features of COVID-19 in elderly patients: A comparison with young and middle-aged patients. The Journal of Infection 80:e14-e18. DOI: 10.1016/j.jinf.2020.03.005. 
376 Lorenzo-Redondo R, Nam HH, Roberts SC, Simons LM, Jennings LJ, Qi C, Achenbach CJ,

377 Hauser AR, Ison MG, Hultquist JF, Ozer EA. 2020. A Unique Clade of SARS-CoV-2

378 Viruses is Associated with Lower Viral Loads in Patient Upper Airways. medRxiv: The

379 Preprint Server for Health Sciences. DOI: 10.1101/2020.05.19.20107144.

380 Ozono S, Zhang Y, Ode H, Tan TS, Imai K, Miyoshi K, Kishigami S, Ueno T, Iwatani Y, Suzuki

381 T, Tokunaga K. 2020. Naturally mutated spike proteins of SARS-CoV-2 variants show

382 differential levels of cell entry. bioRxiv:2020.06.15.151779. DOI:

383 10.1101/2020.06.15.151779.

384 Pachetti M, Marini B, Benedetti F, Giudici F, Mauro E, Storici P, Masciovecchio C, Angeletti S,

385 Ciccozzi M, Gallo RC, Zella D, Ippodrino R. 2020. Emerging SARS-CoV-2 mutation hot

386 spots include a novel RNA-dependent-RNA polymerase variant. Journal of Translational 387 Medicine 18:179. DOI: 10.1186/s12967-020-02344-6.

388 Petersen E, Gökengin D. 2020. SARS-CoV-2 epidemiology and control, different scenarios for 389 Turkey. Turk J Med Sci:6.

390 Riou J, Althaus CL. 2020. Pattern of early human-to-human transmission of Wuhan 2019 novel coronavirus (2019-nCoV), December 2019 to January 2020. Eurosurveillance 25. DOI:

Wagner C, Roychoudhury P, Hadfield J, Hodcroft EB, Lee J, Moncla LH, Müller NF, Behrens C, Huang M-L, Mathias P, Pepper G, Shrestha L, Xie H, Neher RA, Baird GS, Greninger AL, Jerome KR, Bedford T. 2020.Comparing viral load and clinical outcomes in Washington State across D614G mutation in spike protein of SARS-CoV-2. Available at https://github.com/blab/ncov-D614G (accessed July 17, 2020). 
398 Walls AC, Park Y-J, Tortorici MA, Wall A, McGuire AT, Veesler D. 2020. Structure, Function, 399 and Antigenicity of the SARS-CoV-2 Spike Glycoprotein. Cell 181:281-292.e6. DOI:

400 10.1016/j.cell.2020.02.058.

401

402

403

404

405

406

407

408

409

410

411

412

413

414

415

416

417

418

419

420

Wong J, Jamaludin SA, Alikhan MF, Chaw L. 2020. Asymptomatic transmission of SARS-CoV2 and implications for mass gatherings. Influenza and Other Respiratory Viruses. DOI: 10.1111/irv.12767.

Worobey M, Pekar J, Larsen BB, Nelson MI, Hill V, Joy JB, Rambaut A, Suchard MA, Wertheim JO, Lemey P. 2020. The emergence of SARS-CoV-2 in Europe and the US. bioRxiv. DOI: 10.1101/2020.05.21.109322.

Yurkovetskiy L, Pascal KE, Tomkins-Tinch C, Nyalile T, Wang Y, Baum A, Diehl WE, Dauphin A, Carbone C, Veinotte K, Egri SB, Schaffner SF, Lemieux JE, Munro J, Sabeti PC, Kyratsous CA, Shen K, Luban J. 2020. SARS-CoV-2 Spike protein variant D614G increases infectivity and retains sensitivity to antibodies that target the receptor binding domain. bioRxiv:2020.07.04.187757. DOI: 10.1101/2020.07.04.187757.

Zhang Y-Z, Holmes EC. 2020. A Genomic Perspective on the Origin and Emergence of SARSCoV-2. Cell 181:223-227. DOI: 10.1016/j.cell.2020.03.035.

Zhang L, Jackson CB, Mou H, Ojha A, Rangarajan ES, Izard T, Farzan M, Choe H. 2020. The D614G mutation in the SARS-CoV-2 spike protein reduces S1 shedding and increases infectivity. bioRxiv:2020.06.12.148726. DOI: 10.1101/2020.06.12.148726.

\section{Figure Legends}

Figure 1. Mutation density has a strong correlation with time in isolates carrying both 14408 C $>$ T and $23403 \mathrm{~A}>\mathbf{G}$ mutations. The top two scatter plots show the distribution of average 
421 synonymous mutations per day, while the bottom two show the same distribution for non-

422 synonymous mutations. The left two plots have the reference nucleotide in base 14408 and 23403 ,

423 and the right two plots have the mutations of interest in both bases. Red dots indicate average

424 density of samples from the UK, blue dots indicate the average density of US samples. The

425 correlation coefficients are calculated using Spearman correlation using the combined sets of both 426 countries.

427 Figure 2. Synonymous mutation density rates do not shift significantly between early and

428 late periods when comparing WT and MT samples. The boxplots show the synonymous

429 mutation density of UK-WT, UK-MT, US-WT, and US-MT samples divided into early period

430 (60-100 days) and late period (101-140 days), with gray bars indicating early period samples,

431 and orange bars indicating late period samples. The average mutation density per day is

432 calculated in average nucleotides mutated per gene locus across all isolates. Mutations in the

433 RdRp coding regions are included in both the RdRp plot and the Orflab plot.

434 Figure 3. MT samples display higher non-synonymous mutation density increases in late

435 period compared to WT samples. The boxplots show the non-synonymous mutation density of

436 UK-WT, UK-MT, US-WT, and US-MT samples divided into early period (60-100 days) and late

437 period (101-140 days), with gray bars indicating early period samples, and orange bars indicating

438 late period samples. The average mutation density per day is calculated in average nucleotides

439 mutated per gene locus across all isolates. Mutations in the RdRp coding regions are included in

440 both the RdRp plot and the Orflab plot.

441 Figure 4. Mutation densities in MT isolates increase more rapidly compared to WT isolates

442 after first identified MT genome. A) bar plots for isolates in the 60-80 day time period. B) bar

443 plots for isolates in the 121-140 day time period. The bar plots show 100 nucleotide long bins 
444 indicating regions on the genome in the x-axis, and the total number of isolates with mutations in

445 the indicated regions in the y-axis, with green bars indicating WT isolates and orange bars

446 indicating MT isolates. The top two bar plots for each subfigure indicate synonymous mutations,

447 with the bottom two indicating non-synonymous mutations. The left bar plots show the RdRp

448 coding region, while the right bar plots show the $\mathrm{S}$ gene.

449

450 


\section{Figure 1}

Mutation density has a strong correlation with time in isolates carrying both $14408 \mathrm{C}>\mathrm{T}$ and $23403 \mathrm{~A}>\mathrm{G}$ mutations.

The top two scatter plots show the distribution of average synonymous mutations per day, while the bottom two show the same distribution for non-synonymous mutations. The left two plots have the reference nucleotide in base 14408 and 23403, and the right two plots have the mutations of interest in both bases. Red dots indicate average density of samples from the UK, blue dots indicate the average density of US samples. The correlation coefficients are calculated using Spearman correlation using the combined sets of both countries. 

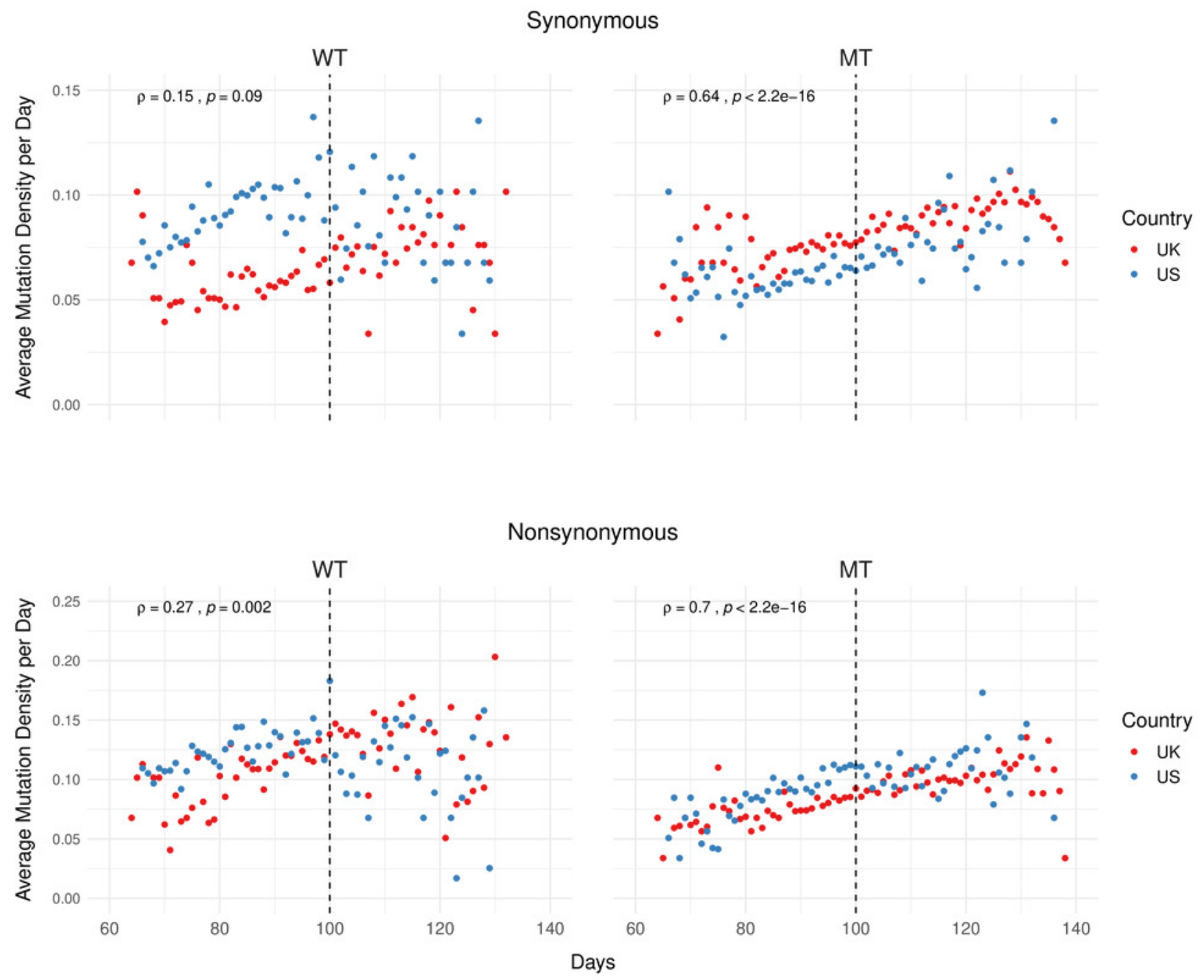


\section{Figure 2}

Synonymous mutation density rates do not shift significantly between early and late periods when comparing WT and MT samples.

The boxplots show the synonymous mutation density of UK-WT, UK-MT, US-WT, and US-MT samples divided into early period (60-100 days) and late period (101-140 days), with gray bars indicating early period samples, and orange bars indicating late period samples. The average mutation density per day is calculated in average nucleotides mutated per gene locus across all isolates. Mutations in the RdRp coding regions are included in both the RdRp plot and the Orf1ab plot. 
S

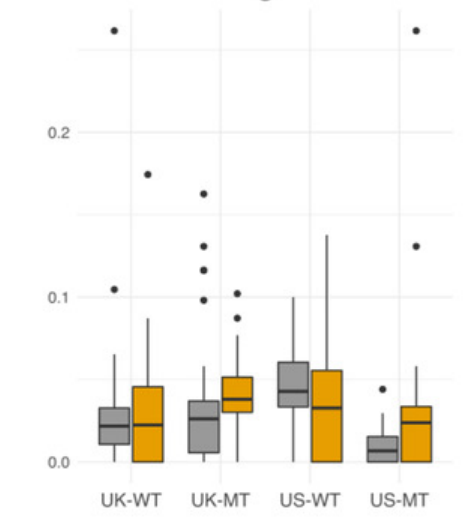

N

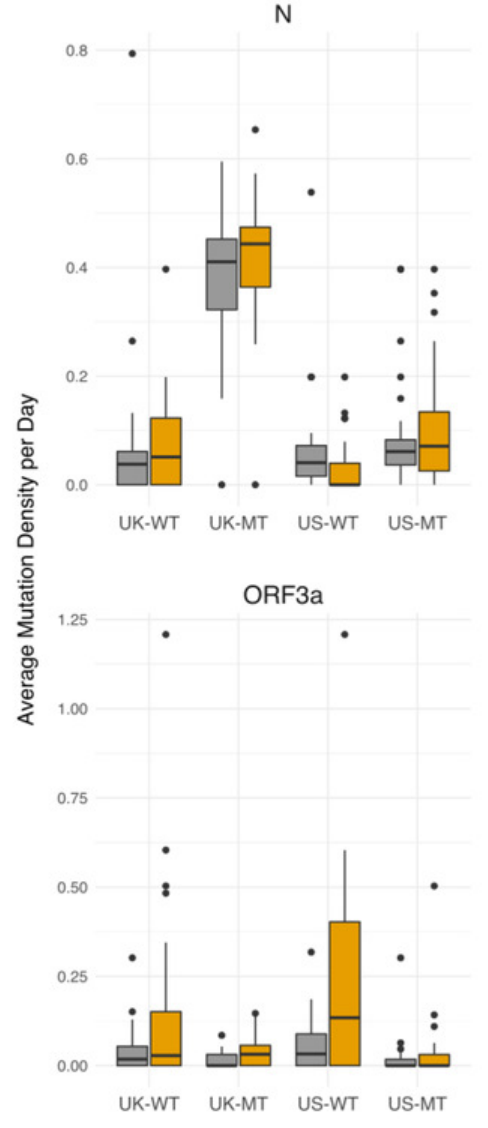

ORF7b
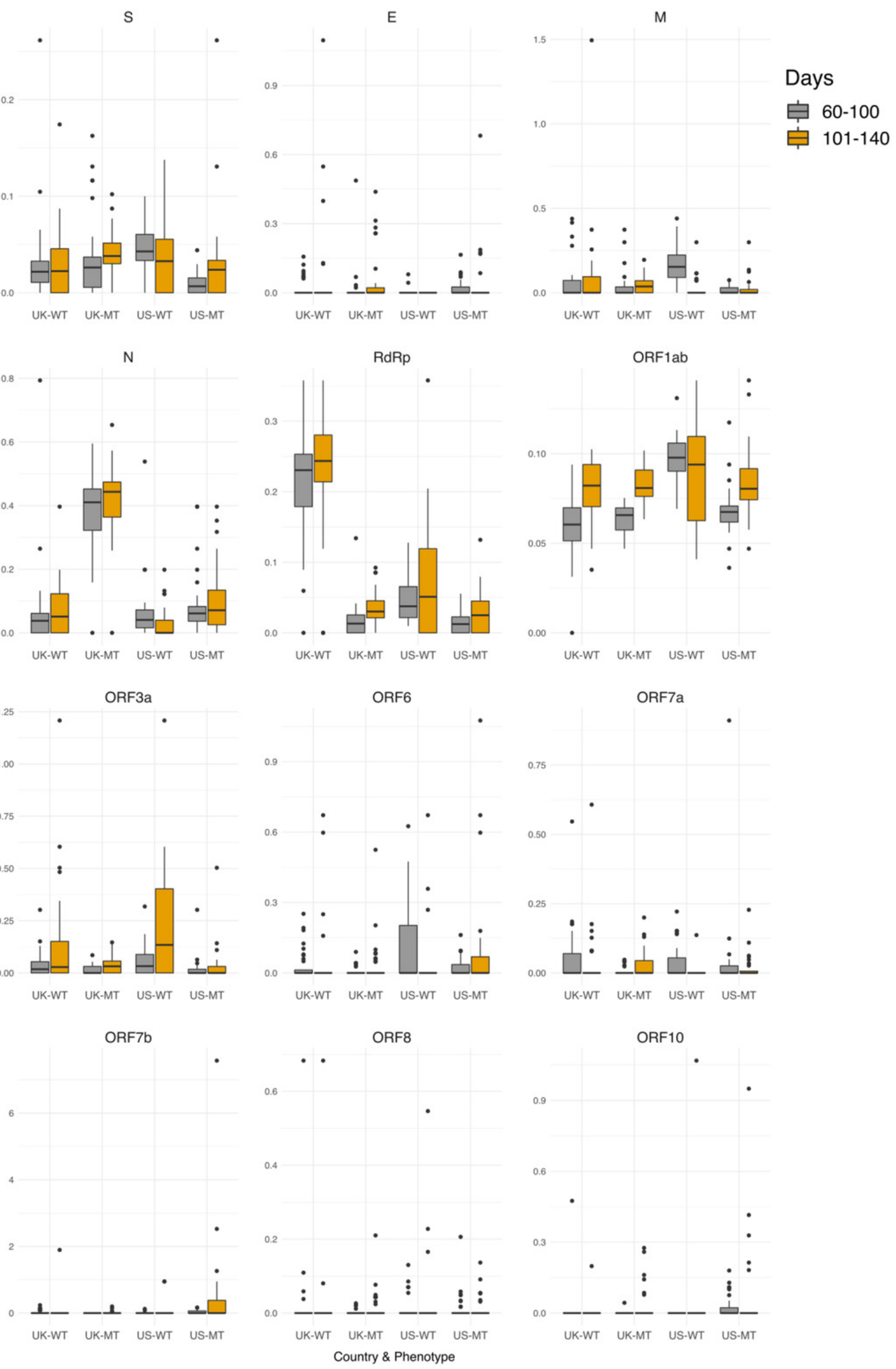


\section{Figure 3}

MT samples display higher non-synonymous mutation density increases in late period compared to WT samples.

The boxplots show the non-synonymous mutation density of UK-WT, UK-MT, US-WT, and USMT samples divided into early period (60-100 days) and late period (101-140 days), with gray bars indicating early period samples, and orange bars indicating late period samples. The average mutation density per day is calculated in average nucleotides mutated per gene locus across all isolates. Mutations in the RdRp coding regions are included in both the RdRp plot and the Orf1ab plot. 


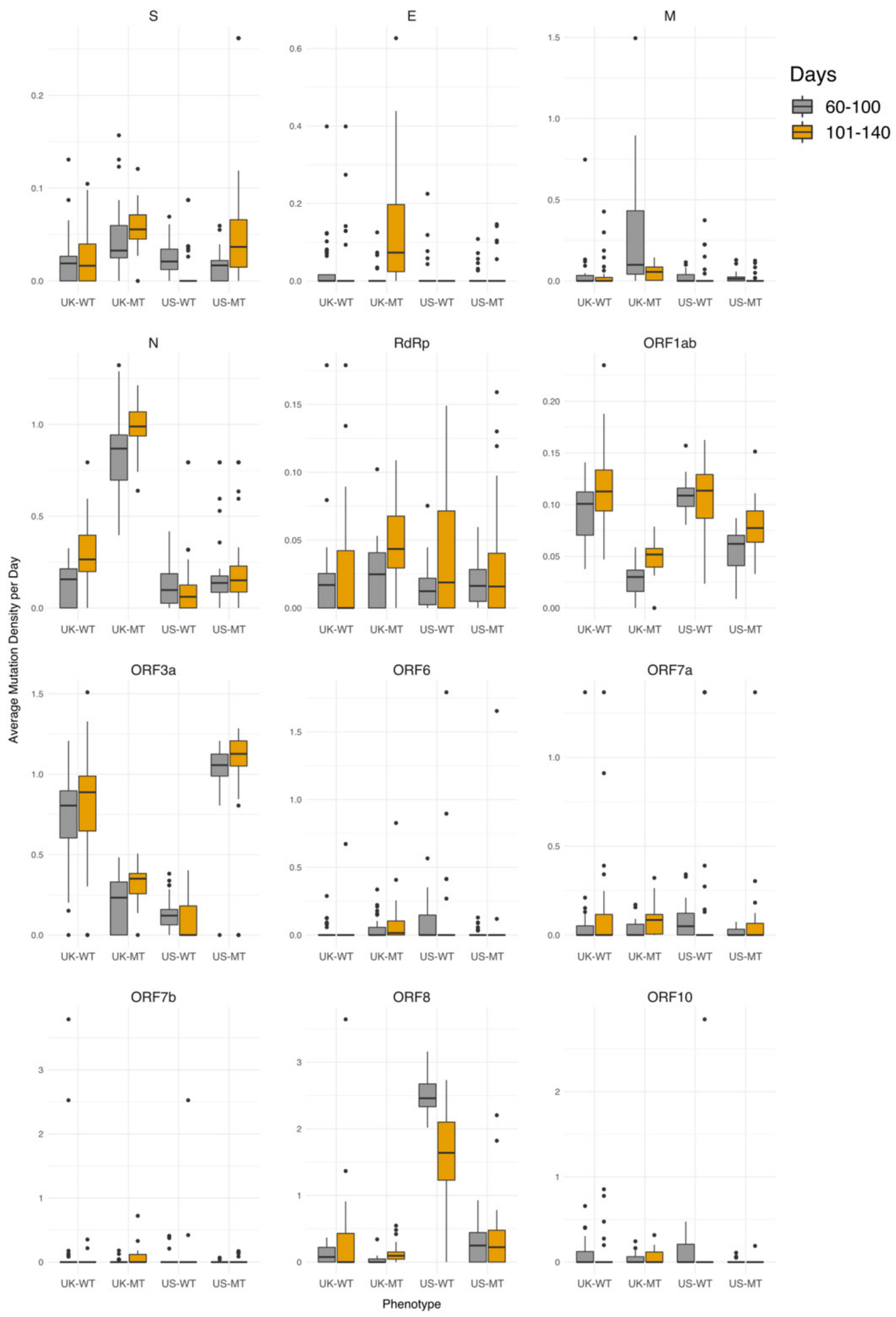




\section{Figure 4}

Mutation densities in MT isolates increase more rapidly compared to WT isolates after first identified MT genome.

A) Barplots for isolates in the 60-80 day time period. B) Barplots for isolates in the 121-140 day time period. The barplots show 100 nucleotide long bins indicating regions on the genome in the x-axis, and the total number of isolates with mutations in the indicated regions in the $y$-axis, with green bars indicating WT isolates and orange bars indicating MT isolates. The top two barplots for each subfigure indicate synonymous mutations, with the bottom two indicating non-synonymous mutations. The left barplots show the RdRp coding region, while the right barplots show the $\mathrm{S}$ gene. 
A.

Days 60-80
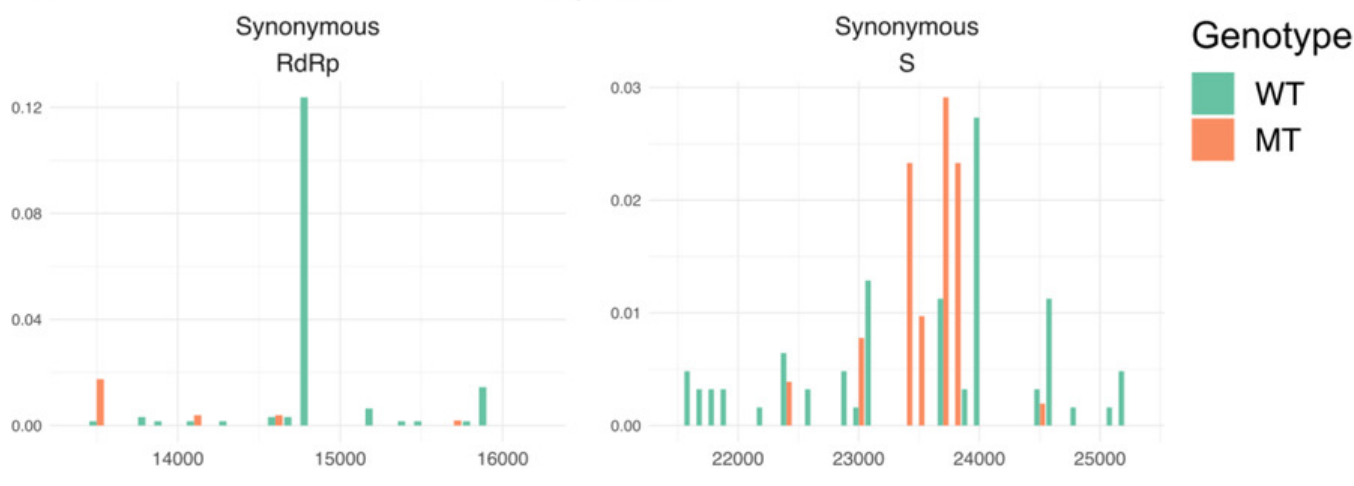

Nonsynonymous

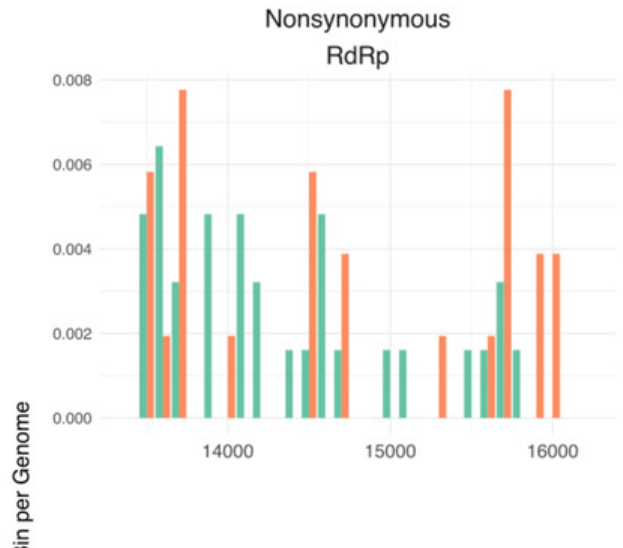

Nonsynonymous

$\mathrm{S}$

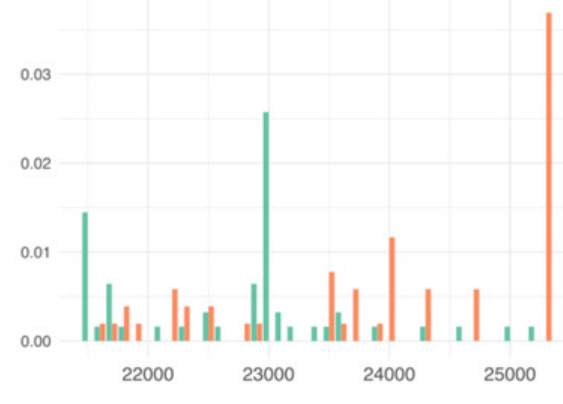

B.

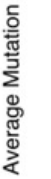
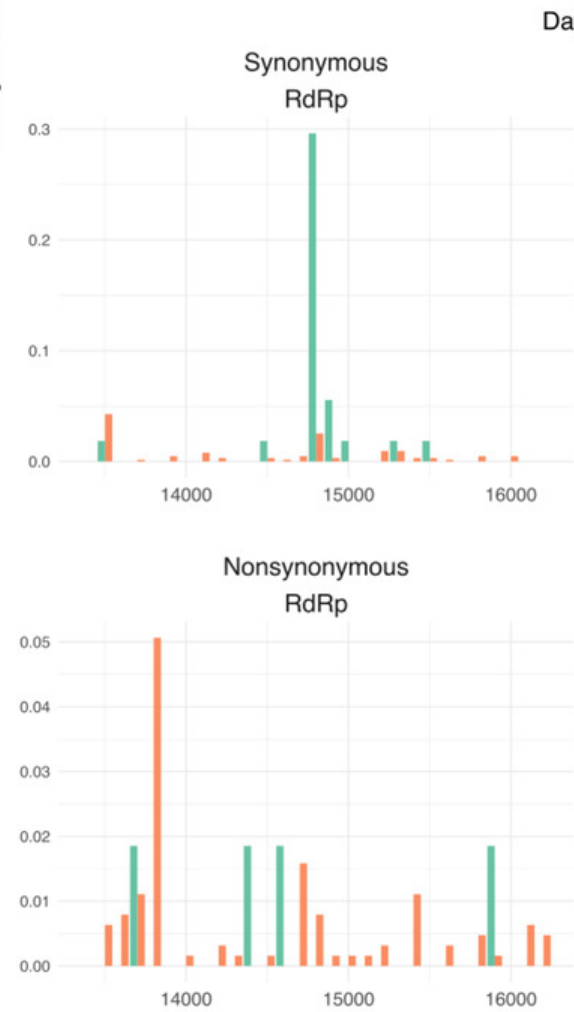

Days 121-140
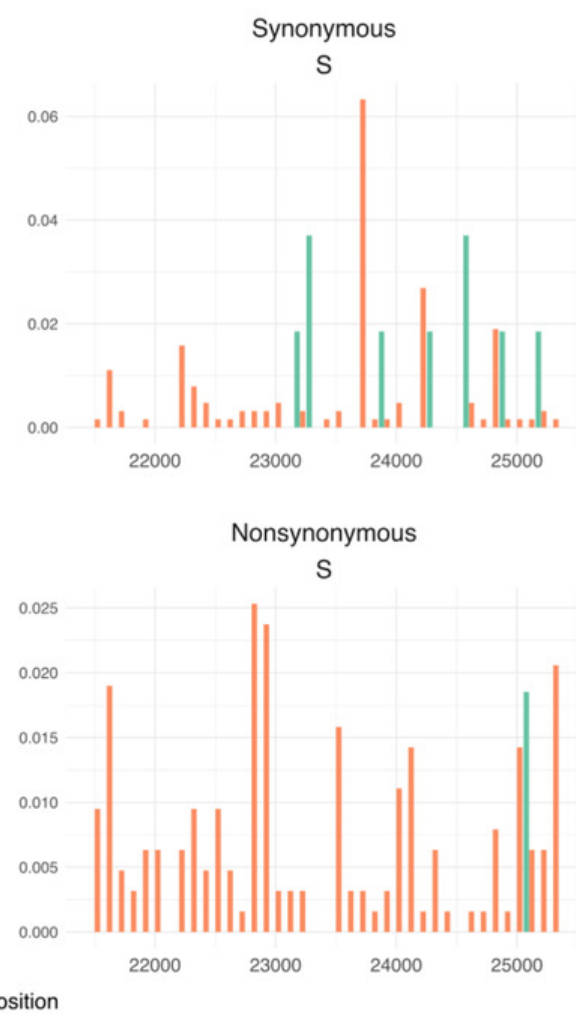